\title{
Роль малых дыхательных путей при бронхиальной астме
}

ФГУ "НИИ пульмонологии" ФМБА России: 105077, Москва, ул. 11-я Парковая, 32, к. 4

\section{S.N.Avdeev \\ A role of small airways in bronchial asthma}

Key words: bronchial asthma, small airways, distal airways, extra-fine inhaled steroids, combined drugs.

Ключевые слова: бронхиальная астма, малые дыхательные пути, дистальные дыхательные пути, экстрамелкодисперсные ингаляционные глюкокортикостероиды, комбинированные препараты.

Бронхиальная астма (БА) относится к числу наиболее часто встречаемых хронических заболеваний легких, общая распространенность астмы в настоящее время составляет около $10 \%$ от общей популяции [1]. Заболеваемость БА постоянно возрастает и вносит существенный вклад в повышение болезненности и общее бремя расходов на здравоохранение современного общества. В настоящее время БА рассматривается как хроническое воспалительное заболевание дыхательных путей (ДП), которое присутствует у больных с любой степенью тяжести заболевания, даже у пациентов со впервые выявленной БА [2]. В соответствие с национальными и международными рекомендациями по ведению больных БА, основной задачей терапии является достижение и поддержание оптимального контроля над заболеванием, главным образом, за счет подавления воспаления в ДП, что, в свою очередь, приводит к снижению числа обострений [2].

Ингаляционные глюкокортикостероиды (иГКС) сегодня являются наиболее эффективными из известных противовоспалительных лекарственных средств для терапии БА и рекомендованы в качестве терапии 1-й линии для достижения контроля над персистирующей БА [2]. В случае недостаточного контроля с помощью монотерапии иГКС рекомендовано дальнейшее повышение дозы иГКС или добавление к ним длительно действующих $\beta_{2}$-агонистов (ДДБА) [2]. Однако, несмотря на терапию максимальными дозами иГКС с добавлением ДДБА или без них, у 20-50 \% пациентов с БА различной степени тяжести не удается достичь хорошего контроля над заболеванием, а 50-85\% больных не в состоянии достичь полного контроля (т. е. состояния, при котором отсутствуют дневные симптомы, ночные пробуждения, нет потребности в использовании короткодействующих $\beta_{2}$-агонистов, в визитах скорой помощи и т. д.) [3]. Кроме того, в длительных проспективных исследованиях было показано, что у больных БА, несмотря на терапию иГКС, скорость снижения функциональных показателей происходит быстрее, по сравнению со здоровыми людьми того же возраста [4]. И наконец, несмотря на то, что тяжелая астма составляет приблизительно $10 \%$ от случаев БА, на терапию таких больных приходится до
30 \% всех расходов на заболевание (главные расходы незапланированные визиты и госпитализация больных), что также связано с недостаточным достижением контроля над БА [5, 6].

Есть несколько причин такой не очень обнадеживающей ситуации, среди которых необходимо отметить и недостатки существующих ингаляционных препаратов, которые не способны одинаково эффективно подавлять воспаление во всех отделах ДП, особенно - в малых ДП, которые сегодня рассматриваются как основное место патологических изменений при БА, и куда данные препараты проникают очень плохо [7-9].

\section{Анатомия и физиология малых ДП}

Трахея делится на 2 главных бронха, каждый из которых в области корней входит в легкие. После вхождения в легкие бронхи многократно и последовательно разветвляются на более мелкие, что приводит к значительному увеличению числа воздухоносных путей и прогрессивному уменьшению их диметра [10].

К бронхиолам относят ДП диаметром < 2-3 мм, не имеющие хрящевых колец и слизистых желез [11]. Каждая терминальная (мембранозная) бронхиола разделяется на 2 или более респираторные бронхиолы, которые переходят в альвеолярные ходы, затем в альвеолярные мешочки, и наконец, в альвеолы. В состав каждой вторичной легочной дольки входит от 4 до 8 терминальных бронхиол с соответствующими им первичными дольками (ацинусами) [12]. Стенка терминальных бронхиол содержит гладкомышечные клетки, а стенка респираторных - реснитчатые эпителиальные клетки и альвеолоциты и не имеет гладкомышечных клеток.

В настоящее время к малым (дистальным) относят ДП диаметром < 2 мм (что соответствует 6-7 генерациям воздухоносных путей по классификации Weibel) [10], куда входят терминальные и респираторные бронхиолы, а также альвеолярные ходы. Терминальные бронхиолы относятся к воздухопроводящим (кондуктивным) ДП, а респираторные бронхиолы и альвеолярные ходы - к переходным ДП, т. е. они принимают участие в проведении воздуха и газообмене. 


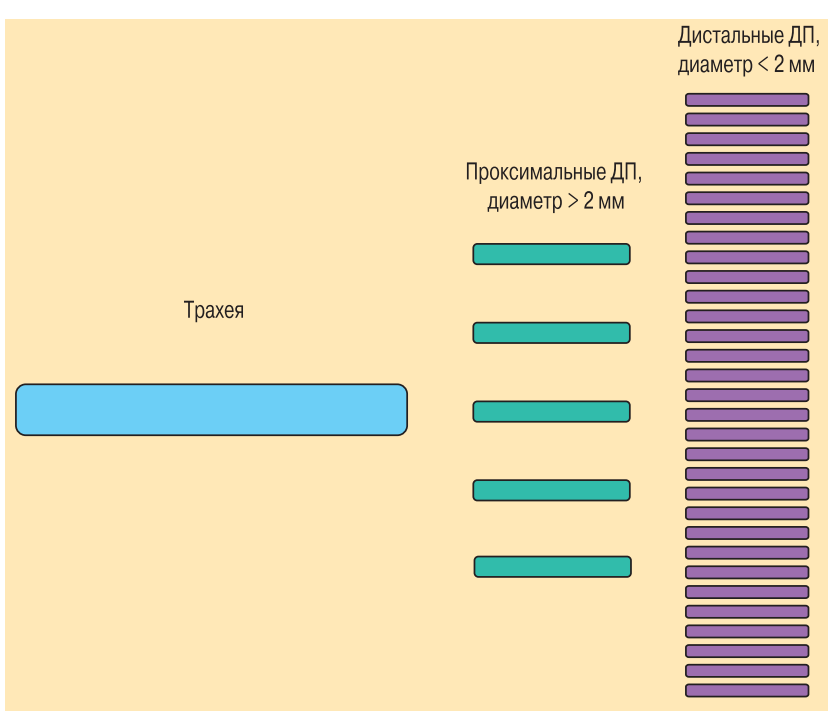

Рис. 1. Общая площадь ДП увеличивается по мере уменьшения их диаметра

Увеличение числа воздухоносных путей многократно превосходит снижение их диметра, поэтому общая поперечная площадь дистальных ДП очень велика. Так, общая площадь сечения малых ДП (53-186 $\left.\mathrm{cm}^{3}\right)$ во много раз превышает площадь трахеи (3-4 см³) и крупных бронхов (4-10 см³) (рис. 1). Поэтому малые ДП вносят лишь небольшой вклад в общее сопротивление бронхиального дерева [10]. В дополнение к этому нужно отметить, что малые ДП находятся внутри соединительнотканного каркаса легких, что позволяет передавать напряжение от легочной паренхимы к дистальным ДП. При повышении легочных объемов возрастает напряжение стенок малых ДП, что приводит к увеличению их диаметра. В свою очередь, это ведет к снижению сопротивления ДП при высоких легочных объемах. Любые патологические изменения в стенке малых ДП (отек, воспаление и др.) "приглушают" передачу напряжения к ним от легочной паренхимы, что будет способствовать коллапсу дистальных ДП, особенно при низких легочных объемах. Во время нормального спокойного дыхания на уровне функциональной остаточной емкости (FRC) малые ДП вносят от 10 до $20 \%$ в общее сопротивление ДП [13,14]. Важно отметить, что измеряемое сопротивление зависит от частоты, при которой происходит измерение. Низкие частоты более эффективно достигают малых ДП, по сравнению с высокими частотами, и демонстрируют больший вклад дистальных ДП в общее легочное сопротивление [15]. В нормальных легких воздушный поток в малых ДП - ламинарный, а в центральных ДП - турбулентный. В таблице суммированы наиболее важные отличия между малыми (дистальными) и крупными (проксимальными) ДП.

\section{Физиологическая оценка функции малых ДП}

Функциональная оценка поражения малых ДП достаточно сложна и требует использования специальных методов исследования [7]. Бронхоскопические методы для прямой оценки сопротивления ДП - ca- мые точные, но их по-прежнему используют только в научных исследованиях, т. к. они являются инвазивными и достаточно трудоемкими [16-18].

Характерными признаками обструкции малых ДП являются их преждевременное экспираторное закрытие и развитие воздушной ловушки, регионарная гетерогенность и повышенная зависимость ограничения воздушного потока от величины легочных объемов. Показатели, направленные на выявление и оценку данных изменений, могут рассматриваться как полезные суррогатные показатели функции малых ДП.

В присутствии преждевременного экспираторного закрытия малых ДП и воздушной ловушки происходит повышение остаточного объема (RV) [19]. Так как общая емкость легких (TLC) обычно повышена при обструктивных болезнях легких, то соотношение RV / TLC является лучшим показателем легочной гиперинфляции [20]. Из показателей спирометрии наиболее чувствительными показателями гиперинфляции являются параметры жизненной (VC) или форсированной жизненной емкости легких (FVC) и емкости вдоха (IC) [21], которые относительно хорошо корелируют с показателем RV / TLC (у детей FVC плохо коррелирует с RV / TLC, что, возможно, связано с неточностью должных величин у растущего организма). По мнению некоторых экспертов, отношение форсированной VC к медленной VC (FVC / SVC) также является маркером поражения малых ДП [7, 21].

Показатель средней объемной скорости выдоха на уровне $25-75$ \% FVC ( $\left.\mathrm{FEF}_{25-75 \%}\right)$ является спирометрическим параметром, который наиболее часто цитируется как индикатор обструкции малых ДП [22]. Как маркер обструкции ДП, $\mathrm{FEF}_{25-75 \%}$ коррелирует с отношением объема форсированного выдоха за 1-ю с - FEV 1 / FVC (\% от должных), но нелинейно. Так, при легкой обструкции $\mathrm{FEF}_{25-75 \%}$ снижается в большей степени, чем $\mathrm{FEV}_{1}$ / FVC. Показатели форсированных экспираторных потоков на уровне средних и низких легочных объемов подвержены высокой вариабельности и зависят от функции крупных ДП и легочных объемов [23]. К недостаткам $\mathrm{FEF}_{25-75 \%}$ можно отнести и его плохую корреляцию с параметрами легочной гиперинфляции (FVC и RV / TLC) [24].

Регионарная гетерогенность сопротивления ДП лучше всего выявляется с помощью методов вымывания азота и имидж-диагностики. Уровень наклона

Таблица

Различия между проксимальными и дистальными ДП

\begin{tabular}{|c|c|}
\hline Проксимальные ДП & Дистальные ДП \\
\hline Диаметр > 2 мм & Диаметр < 2 мм \\
\hline Общая площадь небольшая & Общая площадь большая \\
\hline 80-90 \% от общего сопротивления & $10-20$ \% от общего сопротивления \\
\hline Турбулентный поток & Ламинарный поток \\
\hline $\begin{array}{l}\text { Не участвуют в газообмене (анато- } \\
\text { мическое мертвое пространство) }\end{array}$ & Участвуют в газообмене \\
\hline
\end{tabular}


альвеолярного плато в тесте с вымыванием азота $\left(\mathrm{dN}_{2}\right)$ отражает преимущественно патологию дистальных ДП, однако также зависит от равномерности распределения вентиляции и обструкции крупных бронхов [25]. Объем закрытия (CV) является более специфичным показателем, отражающим преждевременное экспираторное закрытие ДП, хорошо коррелирует с RV / TLC и характеризуется большей чувствительностью для выявления дистальной обструкции, чем все другие известные параметры. Однако CV также является довольно вариабельной переменной [26, 27], и бывает сложно определить точку изгиба (inflection point) на кривой вымывания азота, если наклон кривой достаточно крутой. Еще одним чувствительным тестом для оценки малых ДП является различие среднепотоковых показателей при вдыхании воздуха и гелиокса $\left(\Delta \mathrm{V}_{\max 50}\right)$, данный параметр применялся для выявления разных типов ответа на бронхолитики [28], однако ценность теста также страдает из-за его высокой интериндивидуальной вариабельности [29, 30].

Перспективным методом для оценки малых ДП является импульсная осциллометрия, которая измеряет частотную зависимость легочного сопротивления и реактанса (reactance - совокупность инерционного и эластического сопротивления дыхательной системы) [31, 32], но данная технология еще требует дальнейшей валидизации.

Среди новых функциональных методов оценки малых ДП можно также отметить тест альвеолярной концентрации оксида азота (CalvNO), который, по данным первых исследований, хорошо коррелирует с $\mathrm{FEF}_{25-75 \%}$ и параметрами метода вымывания азота $[33,34]$.

\section{Рентгенологические методы оценки малых ДП}

Современные методы имидж-диагностики, такие как компьютерная томография высокого разрешения (КТВР), позволяют "рассмотреть" ДП диамет- ром > 2 мм [35]. Однако при наличии изменений в малых ДП возможна их визуализация, это т. н. прямые признаки поражения дистальных ДП: плохо очерченные центрилобулярные узелки и мелкие разветвленные затемнения (branching opacities), отражающие перибронхиальные утолщения и слизистые пробки в бронхиолах [36, 37]. С другой стороны, оценка малых ДП возможна с помощью непрямых КТ-признаков, отражающих изменения, связанные с формированием воздушных ловушек - регионов с низкой плотностью паренхимы (рис. 2) [38, 39]. Эти КТ-признаки являются более чувствительными, по сравнению со спирометрическими, для выявления ранних изменений в дистальных ДП у больных БА легкой степени [40]. Регионарные воздушные ловушки приводят к формированию мозаичного паттерна олигоэмии, имеющего "пятнистый" или "географический" тип распространения, особенно хорошо выявляемый во время выдоха [37, 41]. На выдохе области паренхимы легких, соответствующие неизмененным бронхиолам, становятся более плотными, в то время как пораженные сегменты остаются сверхпрозрачными, т. к. обструкция на уровне малых ДП препятствует полной эвакуации воздуха, т. е. ведет к развитию воздушных ловушек. Необходимо подчеркнуть, что КТ-признаки воздушных ловушек и мозаичной олигоэмии могут быть обнаружены не только при БА, но и при других заболеваниях, для которых характерно поражение малых ДП, например при облитерующем бронхиолите [42].

По данным исследования T. Ueda et al., изменения легочной плотности при разных объемах легких имеют хорошую корреляционную взаимосвязь с показателями, отражающими функцию дистальных ДП, и с тяжестью БА [43]. По данным другого исследования, снижение плотности легких у больных астмой хорошо коррелирует с параметрами RV / TLC и осцилляторным сопротивлением ДП [44]. Y.M.Lee et al. показали, что прямые признаки поражения малых ДП в виде центрилобулярных узелков достаточно

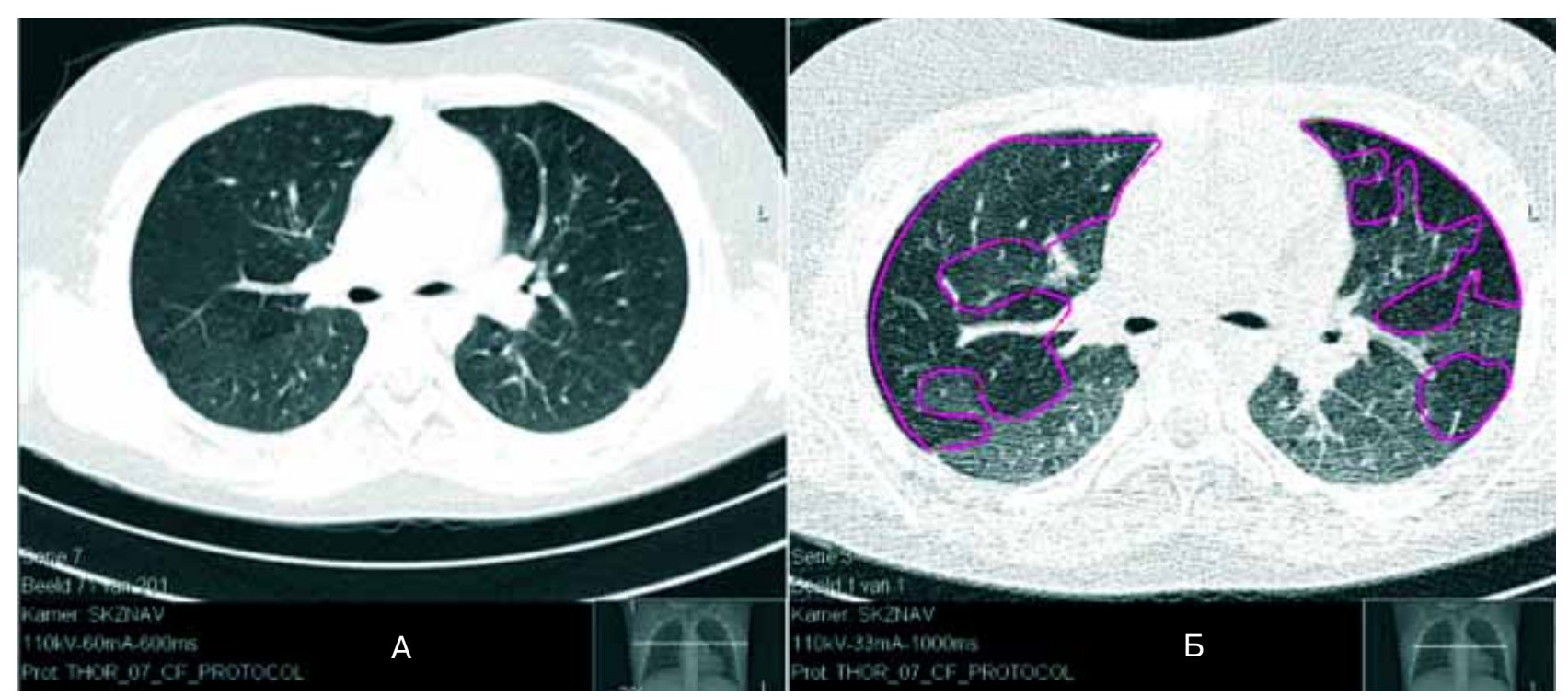

Рис. 2. Оценка воздушной ловушки с помощью КТВР: А - на вдохе; Б - на выдохе

Примечание: обведенный контур включает в себя регионы с пониженной плотностью паренхимы легких. 
часто присутствуют у больных с околофатальной астмой, и эти изменения уменьшаются при успешной терапии обострения БА [45].

Регионарные воздушные ловушки могут быть оценены не только качественно, но и количественно, например, с помощью анализа кривых легочной плотности, которые отражают распространение регионов воздушных ловушек в паренхиме легких [40]. Такой количественный анализ воздушных ловушек с помощью КТВР является перспективным методом изучения эффективности лекарственных средств у больных БА [46].

Среди перспективных методов изучения функции малых ДП следует отметить магнитно-резонансную томографию во время ингаляции гиперполяризованного газа, например гелия [47, 48]. Данная технология позволяет выявлять и количественно оценивать гетерогенность вентиляции и перфузии, связанные с регионарными изменениями дистальных ДП [49, 50]. В отличие от КТВР, магнитно-резонансные методики не сопряжены с радиационной нагрузкой на больного и могут быть повторены многократно. Однако поляризация газа является технические сложной и дорогостояшей процедурой, поэтому данный метод пока используется лишь в единичных центрах.

\section{Морфологические изменения малых ДП при БА}

Воспалительные изменения бронхиального дерева при БА, оцениваемые с помощью методов эндобронхиальной биопсии и бронхоальвеолярного лаважа, отражают, прежде всего, изменения в крупных ДП. Доступ к малым ДП с помощью бронхоскопических методик ограничен вследствие их малого диаметра (<2 мм) [51]. Однако уже в 1920-х гг. при проведении аутопсийных исследований было выявлено, что при БА воспаление диффузно поражает все отделы ДП, как центральные, так и дистальные [52].

M.Saetta et al. обнаружили у больных, умерших от БА, выраженные изменения в малых ДП: их обтура-

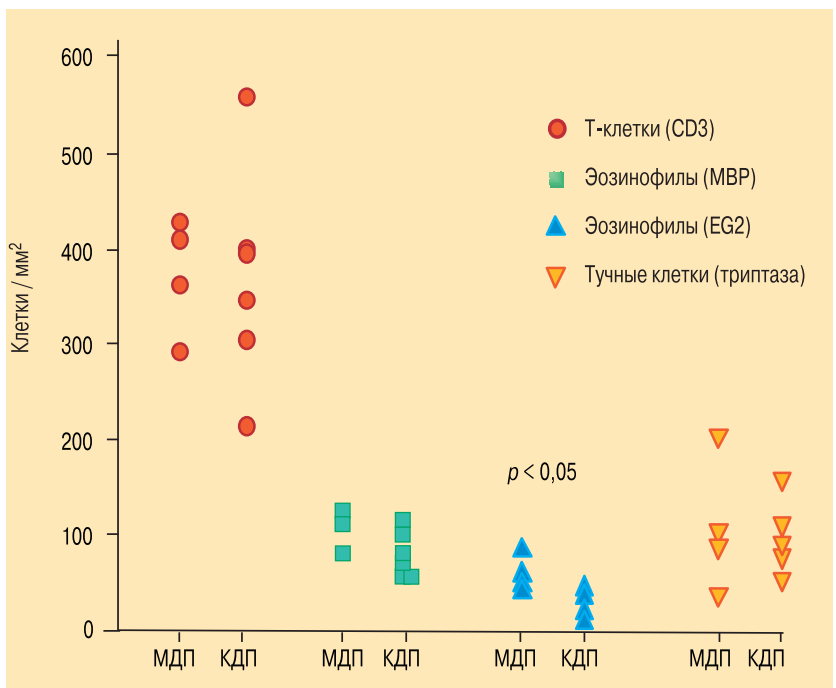

Рис. 3. Воспалительные клетки в крупных (КДП) и малых (МДП) дыхательных путях у больных БА [61] цию слизистыми пробками, утолщение мышечной оболочки и воспалительную инфильтрацию мононуклеарными клетками и эозинофилами [53]. K.Kuwano et al. продемонстрировали выраженное утолщение подслизистого слоя, мышечного слоя и адвентиции малых ДП у пациентов как с фатальной, так и легкой астмой, и данные изменения были более выражены, чем у больных ХОБЛ [54]. Также в аутопсийных исследованиях удалось показать, что наружная оболочка малых ДП в наибольшей степени вовлечена в процесс воспаления и экстрацеллюлярного ремоделирования, в то время как в центральных ДП наибольшие изменения наблюдаются во внутренней оболочке ДП [55-58]. При фатальной астме было выявлено, что некоторые воспалительные и структурные изменения малых ДП распространяются на периваскулярную зону и перибронхиальные альвеолы, что приводит к нарушению поддержки (attachments) альвеол [59, 60]. Данные виды повреждений вносят большой вклад в развитие функциональных нарушений, наблюдаемых на уровне малых ДП, такие как раннее спадение ДП и потеря эластической отдачи [59].

Прижизненные исследования ДП у больных БА очень немногочисленны, т. к. при астме биопсия проводится очень редко. В некоторых центрах проводились морфологические исследования препаратов трансбронхиальной биопсии или тканей легких, полученных при хирургической резекции легких по поводу других заболеваний (не астмы). Данные исследования позволили получить важнейшую информацию о вовлечении малых ДП в патологический процесс, особенно при тяжелой БА. В исследованиях Q.Hamid et al. было выявлено, что у больных БА воспалительный инфильтрат из Т-лимфоцитов и эозинофилов присутствует во всех отделах трахеобронхиального дерева, однако активированные EG2+ эозинофилы преобладают в дистальных ДП (рис. 3), там же отмечаетя более выраженная экспрессия матричной РНК для интерлейкина-5 (IL-5) и эотаксина [61, 62]. И наконец, в еще одном исследовании, при сравнении картины эндобронхиальных биопсий крупных бронхов и трансбронхиальных биопсий (которые больше отражают изменения в малых ДП), M.Kraft et al. обнаружили, что в ранние утренние часы эозинофильное воспаление в дистальных ДП выражено больше, чем в проксимальных, особенно у больных с ночной астмой [63].

\section{Физиологические свидетельства роли малых ДП при БА}

Вовлечение в процесс воспаления малых ДП при БА было убедительно показано в "физиологических" исследованиях. Около 30 лет назад E.R.McFadden et al. обнаружили, что при терапии больных с тяжелым обострением БА высокими дозами небулизированных $\beta_{2}$-агонистов происходит быстрое восстановление функциональных параметров, отражающих проходимость крупных ДП (бронхиальное сопротивление и максимальный форсированный экспира- 


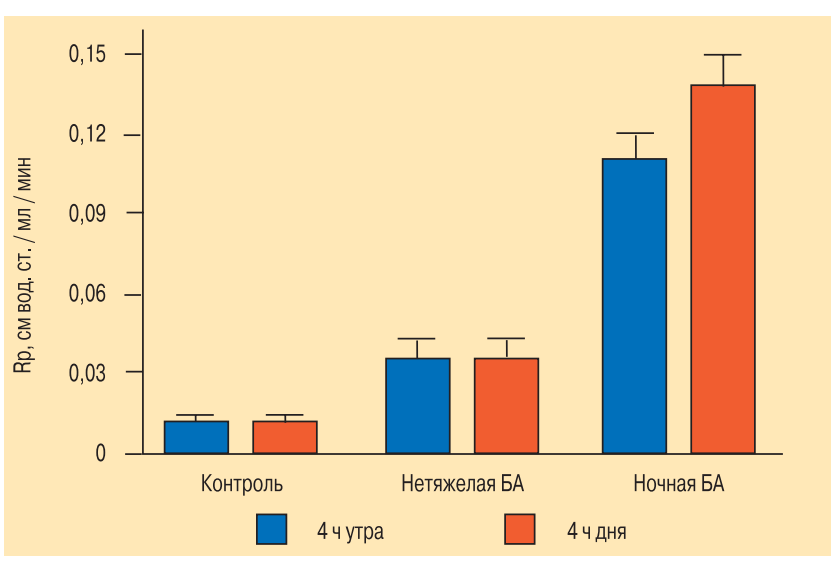

Рис. 4. Сопротивление дистальных ДП (Rp) при тяжелой (ночной) и нетяжелой БА [65]

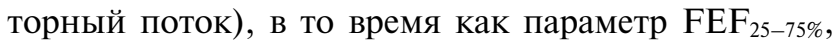
отражающий функцию малых ДП, очень слабо меняется даже после исчезновения всех симптомов и признаков обострения БА [64]. Авторы предположили, что патология малых ДП при БА является клинически скрытой и относительно резистентной к терапии, и может являться предрасполагающим фактором для развития обострений астмы.

Относительно недавно E.M.Wagner et al. доказали значимые изменения малых ДП даже у больных с легкой БА с нормальными показателями спирографии и бронхиального сопротивления по данным бодиплетизмографии [16]. При заклинивании бронхоскопа в ДП у больных БА авторы продемонстрировали, что сопротивление дистальных ДП в 7 раз выше, чем у здоровых добровольцев, что еще раз подтвердило справедливость положения о малых ДП как о "немой" зоне трахеобронхиального дерева. Эта же группа авторов показала, что в ответ на введение гистамина у больных легкой БА происходит драматическое повышение сопротивления дистальных ДП, которое не разрешается полностью с помощью $\beta_{2}$-агонистов [17].

Группа исследователей из Японии, используя метод катетерной микроманометрии, также подтвердила повышение сопротивления малых ДП у больных БА. По их данным, вклад дистальных ДП в общее бронхиальное сопротивление во время вдоха составлял $51 \pm 6 \%$, а во время выдоха $-51 \pm 6 \%$ [18]. В исследовании M.Kraft et al., где была использована бронхоскопическая методика, описанная E.M.Wagner et al., у больных с тяжелой ночной астмой сопротивление в малых ДП, как в дневное, так и в ночное время, превышало показатели больных с нетяжелой астмой в 3,5-3,7 раза, а показатели здоровых людей - в 11-14 раз (рис. 4) [65].

\section{Клиническая значимость роли малых ДП при БА}

В то время как морфологические и функциональные исследования убедительно свидетельствуют о вовлечении малых ДП в патогенез БА, клиническая значимость малых ДП при астме не так очевидна. Важные вопросы, для ответа на которые требуется проведение клинических исследований, сегодня мо-

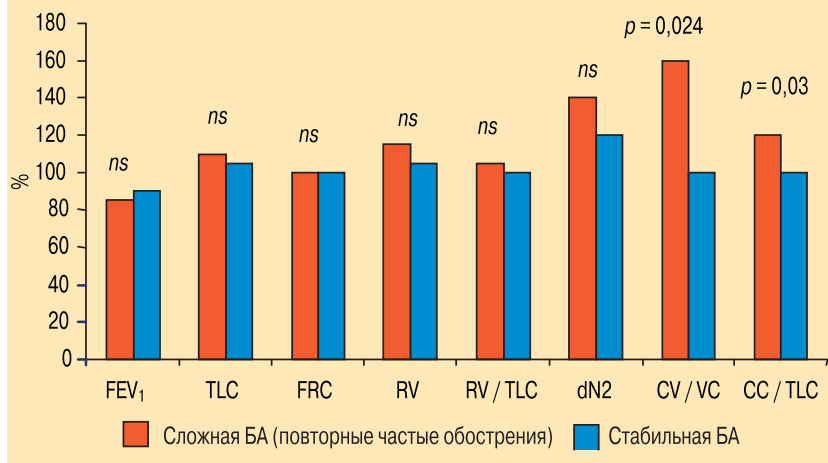

Рис. 5. Ассоциация повторных обострений БА с патологией ДП [68]

гут быть сформулированы так: предрасполагает ли патология малых ДП к развитию обострений и более быстрому прогрессированию бронхиальной обструкции [66-68]?

Некоторые факты подтверждают влияние патологии дистальных ДП на клиническую картину БА. В ретроспективном исследовании J.C. in't Veen et al. обнаружили, что в период ремиссии единственным функциональным показателем, различающим больных БА с частыми и нечастыми обострениями, является объем закрытия (CV) - чувствительный и специфичный физиологический индикатор поражения малых ДП (рис. 5) [68]. Той же группой исследователей было выявлено, что больные с тяжелой стероидо-зависимой астмой имеют более выраженные изменения со стороны дистальных ДП, что нашло отражение у них в более высоких значениях альвеолярной концентрации оксида азота (2,7 ppb vs 0,6 ppb у больных с нетяжелой БА) [69].

В исследовании A.Bourdin et al. больные БА с частыми обострениями отличались от больных с нечастыми обострениями по более выраженному наклону фазы III в тесте вымывания азота (dN2), т. е. по показателю, который также является маркером поражения малых ДП [25]. В этой работе была отмечена достоверная корреляция $\mathrm{dN}_{2}$ с контролем БА $(r=$ $0,62)$, частотой обострений $(r=0,62)$ и соотношением RV / TLC $(r=0,49)$.

Данные наблюдения подтверждают более раннее предположение E.R.McFadden et al., согласно которому персистирующее поражение малых ДП, несмотря на то, что с клинической точки зрения оно практически бессимптомно, может являться предрасполагающим фактором для развития последовательных эпизодов обострения заболевания [64]. Более того, возможно, что более быстрое снижение функциональных показателей у больных БА, по сравнению со здоровыми людьми, также является следствием прогрессирующей обструкции малых ДП, в основе которой лежит их ремоделирование [66, 67]. Вероятнее всего, структурные изменения дистальных ДП, приводящие к фиксированной (необратимой) бронхиальной обструкции, являются результатом плохого подавления воспаления в малых ДП, которые мало доступны для традиционных препаратов ГКС [70]. 


\section{Экстрамелкодисперсные формы ингаляционных} препаратов

Принимая во внимание все эти данные, становится очевидным, что малые ДП должны служить важной мишенью для противовоспалительной и бронхорасширяющей терапии у больных БА. Доставка препарата в ДП зависит от множества факторов, важнейшим из которых является размер частиц лекарственного аэрозоля. Для ингаляционной терапии представляют интерес аэрозольные частицы со среднемассовым аэродинамическим диаметром $(\mathrm{MMAD})<5$ мкм - респирабельные частицы. Чем меньше размер частиц, тем более дистально происходит их депозиция (оседание) [71].

В данном контексте значительный интерес представляют новые препараты иГКС в виде ДАИ с пропеллентом гидрофторалканом (НFA), которые имеют значительно меньший MMAD, по сравнению с традиционными ДАИ на основе фреона (хлорфторуглерода - CFC). Размер частиц беклометазона дипропионата (БДП) в новых ДАИ-НFА оказался значительно меньшим, по сравнению БДП в обычном ДАИ-СFС (1,1 мкм vs 3,5 мкм) [72]. Новые препараты с размерами частиц около 1,5 мкм часто называют "экстрамелкодисперсными" формами аэрозоля.

Изменение профиля частиц аэрозоля привело к изменению величины депозиции препаратов. Так, при использовании экстрамелкодисперсного БДП легочная депозиция у больных БА достигает 56 \%, по сравнению с 4 \% при ингаляции БДП в виде ДАИCFC [70]. Кроме того, сцинтиографические исследования продемонстрировали не только повышение легочной депозиции иГКС > 50 \%, но и преимущественно периферическое распределение препарата в ДП (у больных БА и здоровых добровольцев) [70, 73, 74].

Сравнительные исследования на основе спирометрических данных, показали, что для обеспечения контроля над БА достаточно дозы экстрамелкодисперсного БДП в 2,5-2,6 раза меньше обычной [7581], и при этом практически не наблюдается заметных системных эффектов, при условии, что суточная доза экстрамелкодисперсного БДП не превышает 800 мКг [72].

В нескольких исследованиях была проведена оценка влияния экстрамелкодисперсного БДП на малые ДП [75, 79, 83-86]. Повышение конечноэкспираторных потоковых показателей было отме-
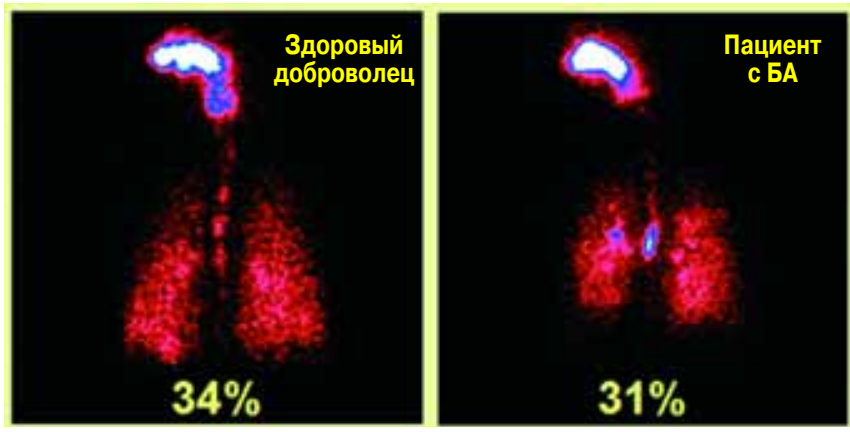

чено при добавлении экстрамелкодисперсного БДП к обычной дозе иГКС у больных с плохо контролируемой БА [83], а также у больных БА, которым впервые в жизни был назначен иГКС (им являлся БДП в виде ДАИ-НFА) [79]. Кроме того, в одном из исследований, в котором для оценки плотности легких использовали КТВР, было показано, что выраженность воздушных ловушек была меньше при терапии экстрамелкодисперсным БДП, по сравнению с БДП в виде ДАИ-СFC [86]. В другом исследовании изучались последствия перевода больных БА с терапии традиционными иГКС (БДП и флутиказона пропионат (ФП) в виде дозированных порошковых ингаляторов) на экстрамелкодисперсный БДП [88]. Несмотря на то, что сравнивались различные иГКС, данные этого исследования позволяют сделать вывод, что экстрамелкодисперсные препараты иГКС способны положительно влиять на функциональные и воспалительные изменения малых ДП, которые адекватно не контролируются с помощью традиционных препаратов.

Можно предположить, что повышение депозиции иГКС может быть связано с повышенным риском развития системных побочных эффектов, например, таких как супрессия продукции кортизола, однако результаты проведенных клинических исследований не подтверждают повышенного риска развития системных эффектов при терапии экстрамелкодисперсным БДП [89].

Относительно недавно появился новый комбинированный препарат в виде ДАИ-НFА, содержащий экстрамелкодисперсный аэрозоль БДП и формотерола (Фостер, 100 / 6 мкг в 1 дозе) [90]. В данном препарате MMAD обеих частей - БДП и формотерола - составляют около 1,3-1,4 мкм [91, 92].

$W$. De Backer et al. провели исследование, в котором изучался профиль депозиции и распределения в легких экстрамелкодисперсной комбинации БДП / формотерол у здоровых добровольцев $(n=8$, средний возраст - 46 лет), у больных БА $(n=8$, средний возраст - 51 год) и у пациентов с ХОБЛ $(n=8$, средний возраст - 61 год) [92]. У здоровых добровольцев средняя легочная депозиция БДП / формотерола составляла $34,1 \pm 9,3$ \% от номинальной дозы, у больных БА - 30,9 $\pm 8,9 \%$, у пациентов с ХОБЛ $33,1 \pm 8,9 \%$ (рис. 6). Отношение центральной и периферической депозиции препарата в легких у больных БА составляло 1,96, при ХОБЛ - 1,94, у здоровых - 1,42. Не было отмечено взаимосвязи между выраженностью бронхиальной обструкции и вели-

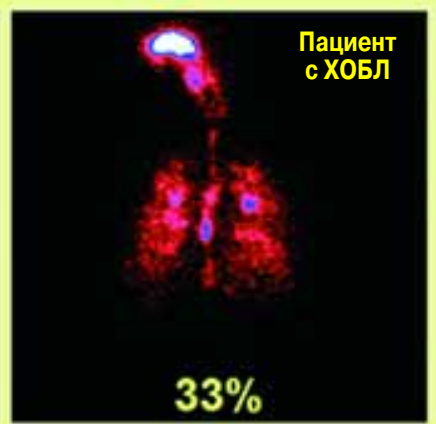

Рис. 6. Легочная депозиция экстрамелкодисперсных частиц комбинации БДП / формотерол у больных БА, ХОБЛ и здоровых добровольцев [92] 
чиной легочной депозиции препарата в легких. Таким образом, экстрамелкодисперсная комбинация БДП / формотерол характеризуется благоприятным профилем доставки в ДП, высокой легочной депозицией и гомогенным распределением препарата во всех отделах ДП, независимо от выраженности бронхиальной обструкции.

Новая экстрамелкодисперная фиксированная комбинация БДП / формотерол оказалась более эффективным препаратом, чем БДП в виде ДАИ-СFC, по влиянию на функциональные показатели, хотя суточные дозы БДП были в 2,5 раза меньше в группе комбинированной терапии [93, 94].

N.Scichilone et al. провели сравнение эффективности терапии экстрамелкодисперсной комбинацией БДП / формотерол в дозе 400 / 24 мкГ в сутки и ФП / салметерол в дозе 500 / 100 мкг в сутки на протяжении 12 нед. у 30 больных БА [95]. Оба вида терапии иГКС / ДДБА привели к значительному приросту $\mathrm{FEV}_{1}$ (в среднем, на 0,37 л), однако гиперреактивность ДП достоверно снизилась только у пациентов, принимавших БДП / формотерол, и только у них отмечена тенденция к снижению CV (почти на 300 мл; $p<0,08$ ) (рис. 7), что является признаком улучшения функции дистальных ДП.

В работах, сравнивавших БДП / формотерол с другими фиксированными комбинациями (ФП / салметерол и будесонид / формотерол), не было выявлено различий между препаратами по влиянию на функциональные и клинические показатели, число обострений и нежелательных реакций [96, 97]. Интересно отметить, что терапия БДП / формотеролом приводила к достоверно бо́льшему увеличению FVC, по сравнению с терапией ФП / салметеролом, что свидетельствует о более "дистальных эффектах" новой фиксированной комбинации [96]. В этих исследованиях номинальные дозы БДП и будесонида или ФП имели соотношение $1: 2,5$, что указывает на то, что новый препарат способен достигать большей эффективности в перерасчете на 1 мг иГКС, по сравнению с традиционными препаратами [96, 97].

В дополнение к этому, недавно опубликованное исследование G.Huchon et al., включавшее в себя 596 пациентов со среднетяжелой и тяжелой БА, продемонстрировало, что экстрамелкодисперсная фиксированная комбинация БДП / формотерол позволяет

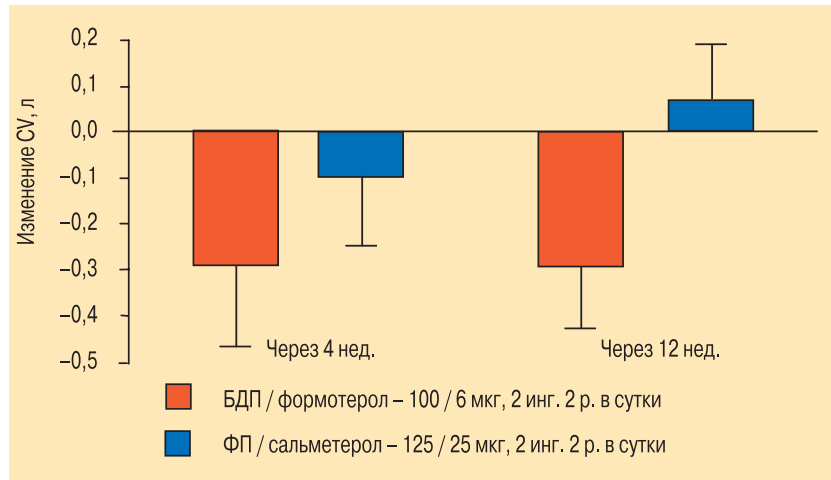

Рис. 7. Влияние комбинированных препаратов БДП / формотерол и ФП / сальметерол на малые ДП у больных БА [95]

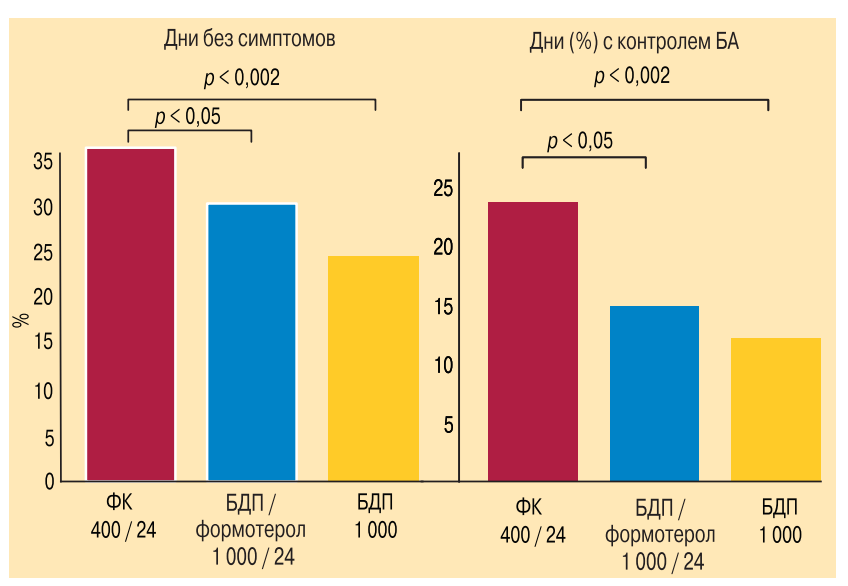

Рис. 8. Преимущества фиксированной комбинации (ФК) БДП / формотерол перед комбинированной терапией теми же компонентами через отдельные ингаляторы [94]

добиться лучшего контроля над астмой, по сравнению со свободной комбинацией неэкстрамелкодисперсными БДП и формотеролом (рис. 8) [94]. Тяжелых обострений БА было достоверно меньше в группе фиксированной комбинации. В данном исследовании доза БДП в комбинации БДП / формотерол была также в 2,5 раза ниже, чем в препаратах сравнения. Более того, терапия экстрамелкодисперсной комбинацией БДП / формотерол привела к снижению подавления оси гипоталамус-гипофизнадпочечники, на что указывало значительное повышение уровня кортизола у больных БА, по сравнению с терапией эквипотентными дозами неэкстрамелкодисперсного БДП + ДДБА [94].

Таким образом, полученные данные делают довольно привлекательной концепцию целевой терапии малых ДП, т. к. сравнительные клинические эффекты терапии могут быть достигнуты при более низких дозах экстрамелкодисперсных иГКС и иГКС / ДДБА, и следовательно, с более низким риском развития нежелательных явлений.

\section{Заключение}

Важная роль патологии малых ДП при астме продемонстрирована с помощью морфологических и физиологических методов исследования, однако клиническое значение данных изменений пока не столь очевидно. Предполагается, что более быстрое, по сравнению с возрастной нормой, прогрессирование бронхиальной обструкции при БА может быть связано с фиксированными (необратимыми) изменениями в малых ДП, т. к. воспаление на этом уровне плохо контролируется современными лекарственными препаратами вследствие их плохой доставки в данные отделы трахеобронхиального дерева. Новые современные ингаляционные препараты иГКС (БДП) и фиксированные комбинации (БДП / формотерол), созданные на основе НFА и имеющие размеры аэрозольных частиц < 1,5 мкм - экстрамелкодисперсные препараты - характеризуются более высокой депозицией в малых ДП, а следовательно, способны обеспечить эффективное подавление воспаления 
и улучшение функции дистальных ДП. Предварительные исследования, изучавшие эффективность новых экстрамелкодисперсных противовоспалительных препаратов, позволяют сделать выводы об их высокой эффективности у больных БА.

\section{Литература}

1. Mannino D.M., Homa D.M., Akinbami L.I. et al. Surveillance of asthma - United States, 1980-1990. Morb. Mort. Wkly Rep. Surveill. Summ. 2002; 51: 1-13.

2. Global initiative for asthma: Global strategy for asthma management and prevention. NHLBI / WHO Workshop Report. Updated 2009. Available at: http://www.ginasthma. com

3. Bateman E.D., Boushey H.A., Bousquet J. et al. Can guideline-defined asthma control be achieved? The gaining optimal asthma control study. Am. J. Respir. Crit. Care Med. 2004; 170: 836-844.

4. Morgan W.J., Stern D.A., Sherrill D.L. et al. Outcome of asthma and wheezing in the first 6 years of life: follow-up through adolescence. Am. J. Respir. Crit. Care Med. 2005; 172: 1253-1258.

5. Van Ganse E., Laforest L., Pietri G. et al. Persistent asthma: disease control, resource utilisation and direct costs. Eur. Respir. J. 2002; 20: 260-267.

6. Van Ganse E., Antonicelli L., Zhang Q. et al. Asthma-related resource use and cost by GINA classification of severity in three European countries. Respir. Med. 2006; 100: 140-147.

7. Burgel P.-R., de Blic J., Chanez P. et al. Update on the roles of distal airways in asthma. Eur. Respir. Rev. 2009; 18: $80-95$.

8. Hyde D.M., Hamid Q., Irvin C.G. Anatomy, pathology, and physiology of the tracheobronchial tree: emphasis on the distal airways. J. Allergy Clin. Immunol. 2009; 124: 72-77.

9. Contoli M., Bousquet J., Fabbri L.M. et al. The small airways and distal lung compartment in asthma and COPD: a time for reappraisal. Allergy 2010; 65: 141-151.

10. Weibel E.R. Morphometry of the human lung. New York: Academic Press; 1963.

11. Wright J.L. Small airway diseases: its role in chronic airway obstruction. Semin. Respir. Med. 1992; 13: 72-84.

12. Garg K., Lynch D.A., Newell J.D., King T.E. Proliferative and constrictive bronchiolitis: classification and radiological features. Am. J. Roentgenol. 1994; 162: 803-808.

13. Macklem P.T., Mead J. Resistance of central and peripheral airways measured by a retrograde catheter. J. Appl. Physiol. 1967, 22: 395-401.

14. Macklem P.T., Mead J. Factors determining maximum expiratory flow in dogs. J. Appl. Physiol. 1968, 25: 159-169.

15. Van Brabandt H., Cauberghs M., Verbeken E. et al. Partitioning of pulmonary impedance in excised human and canine lungs. J. Appl. Physiol. 1983, 55: 1733-1742.

16. Wagner E.M., Liu M.C., Weinmann G.G. et al. Peripheral lung resistance in normal and asthmatic subjects. Am. Rev. Respir. Dis. 1990; 148: 584-588.

17. Wagner E.M., Bleecker E.R., Permutt S. et al. Direct assessment of small airways reactivity in human subjects. Am. J. Respir. Crit. Care Med. 1998; 157: 447-452.

18. Yanai M., Sekizawa K., Ohrui T. et al. Site of airway obstruction in pulmonary disease: direct measurement of intrabronchial partition the central and peripheral airway resistance in a pressure. J. Appl. Physiol. 1992; 72: 1016-1023.

19. Gibson G.J. Pulmonary hyperinflation a clinical overview. Eur. Respir. J. 1996; 9: 2640-2649.
20. Sorkness R.L., Bleecker E.R., Busse W.W. et al. Lung function in adults with stable but severe asthma: air trapping and incomplete reversal of obstruction with bronchodilation. J. Appl. Physiol. 2008; 104: 394-403.

21. Wenzel S.E., Schwartz L.B., Langmack E.L. et al. Evidence that severe asthma can be divided pathologically into two inflammatory subtypes with distinct physiologic and clinical characteristics. Am. J. Respir. Crit. Care Med. 1999; 160: 1001-1008.

22. Marseglia G.L., Cirillo I., Vizzaccaro A. et al. Role of forced expiratory flow at $25-75 \%$ as an early marker of small airways impairment in subjects with allergic rhinitis. Allergy Asthma Proc. 2007; 28: 74-78.

23. Hansen J.E., Sun X.G., Wasserman K. Discriminating measures and normal values for expiratory obstruction. Chest 2006; 129: 369-377.

24. Pellegrino R., Viegi G., Brusasco V. et al. Interpretative strategies for lung function tests. Eur. Respir. J. 2005; 26: 948-968.

25. Bourdin A., Paganin F., Prefaut C. et al. Nitrogen washout slope in poorly controlled asthma. Allergy 2006; 61: 85-89.

26. Make B., Lapp N.L. Factors influencing the measurement of closing volume. Am. Rev. Respir. Dis. 1975; 111: 749-754.

27. McFadden E.R., Holmes B., Kiser R. Variability of closing volume measurements in normal man. Am. Rev. Respir. Dis. 1975; 111: 135-140.

28. Antic R., Macklem P.T. The influence of clinical factors on the site of airway obstruction in asthma. Am. Rev. Respir. Dis. 1976; 114: 851-859.

29. Berend N., Nelson A., Rutland M.B. et al. The maximum expiratory flow-volume curve with air and a low-density gas mixture. An analysis of subject and observer variability. Chest 1981; 80: 23-30.

30. Zack R.T., Solliday N.H., Celik L. et al. Variability of the volume of isoflow. Chest 1981; 79: 269-272.

31. Saadeh C., Goldman M., Gaylor P. Forced oscillation using impulse oscillometry (IOS) detects false negative spirometry in symptomatic patients with reactive airways. J. Allergy Clin. Immun. 2003; 111: 136.

32. Landser F.J., Clement J., Van de Woestijne K.P. Normal values of total respiratory resistance and reactance determined by forced oscillations: influence of smoking. Chest 1982; 81: 586-591.

33. Mahut B., Delacourt C., Zerah-Lancner F. et al. Increase in alveolar nitric oxide in the presence of symptoms in childhood asthma. Chest 2004; 125: 1012-1018.

34. Battaglia S., den Hertog H., Timmers M.C. et al. Small airways function and molecular markers in exhaled air in mild asthma. Thorax 2005; 60: 639-644.

35. King G.G., Muller N.L., Par P.D. Evaluation of airways in obstructive pulmonary disease using high-resolution computed tomography. Am. J. Respir. Crit. Care Med. 1999; 159: 992-1004.

36. Lynch D.A., Newell J.D., Tschomper B.A. et al. Uncomplicated asthma in adults: comparison of CT appearance of the lungs in asthmatic and healthy subjects. Radiology 1993; 188: 829-833.

37. Park J.W., Hong Y.K., Kim C.W. et al. High-resolution computed tomography in patients with bronchial asthma: correlation with clinical features, pulmonary functions and bronchial hyperresponsiveness. J. Investig. Allergol. Clin. Immunol. 1997; 7: 186-192.

38. McNitt-Gray M.F., Goldin J.G., Johnson T.D. et al. Development and testing of image-processing methods for the quantitative assessment of airway hyperresponsiveness 
from high-resolution CT images. J. Comput. Assist. Tomogr. 1997; 21: 939-947.

39. Goldin J.G., McNitt-Gray M.F., Sorenson S.M. et al. Airway hyperreactivity assessment with helical thin-section C. Radiology 1998; 208: 321-329.

40. Goldin J.G., McNitt-Gray M.F. Johnson T. et al. Functional helical high-resolution CT: sensitive test for detecting air trapping due to reversible airway hyperreactivity. Radiology 1995; 197 (P): 437.

41. Laurent F., Latrabe V., Raherison $C$. et al. Functional significance of air trapping detected in moderate asthma. Eur. Radiol. 2000; 10: 1404-1410.

42. Jensen S.P., Lynch D.A., Brown K.K. et al. High-resolution $\mathrm{CT}$ features of severe asthma and bronchiolitis obliterans. Clin. Radiol. 2002; 57: 1078-1085.

43. Ueda T., Niimi A., Matsumoto H. et al. Role of small airways in asthma: investigation using high-resolution computed tomography. J. Allergy Clin. Immunol. 2006; 118: 1019-1025.

44. Jain N., Covar R.A., Gleason M.C. et al. Quantitative computed tomography detects peripheral airway disease in asthmatic children. Pediatr. Pulmonol. 2005; 40: 211-218.

45. Lee Y.M., Park J.S., Hwang J.H. et al. High-resolution CT findings in patients with near-fatal asthma: comparison of patients with mild-to-severe asthma and normal control subjects and changes in airway abnormalities following steroid treatment. Chest 2004; 126: 1840-1848.

46. Goldin J.G.,Tashkin D.P., Kleerup E.C. et al. Comparative effects of hydrofluoroalkane and chlorofluorocarbon beclomethasone dipropionate inhalation on small airways: assessment with functional helical thin-section computed tomography. J. Allergy Clin. Immunol. 1999; 104: 258-267.

47. Yablonskiy D.A., Sukstanskii A.L., Leawoods J.C. et al. Quantitative in vivo assessment of lung microstructure at the alveolar level with hyperpolarized 3He diffusion MRI. Proc. Natl. Acad. Sci. USA 2002; 99: 3111-3116.

48. Samee S., Altes T., Powers P. et al. Imaging the lungs in asthmatic patients by using hyperpolarized helium-3 magnetic resonance: assessment of response to methacholine and exercise challenge. J. Allergy Clin. Immunol. 2003; 111: 1205-1211.

49. Kauczor H.U., Chen X.J., van Beek E.J., Schreiber W.G. Pulmonary ventilation imaged bymagnetic resonance: at the doorstep of clinical application. Eur. Respir. J. 2001; 17: 1008-1023.

50. de Lange E.E., Altes T.A., Patrie J.T. et al. Evaluation of asthma with hyperpolarized helium-3 MRI: correlation with clinical severity and spirometry. Chest 2006; 130: 1055-1062.

51. Holz O., Jorres R.A., Magnussen H. Monitoring central and peripheral airway inflammation in asthma. Respir. Med. 2000; 94 (Suppl. D): 7-12.

52. Huber H.L., Koessle K. The pathology of bronchial asthma. Arch. Intern. Med. 1922; 30: 689-760.

53. Saetta M., DeStefano A., Rosina C. et al. Quantitative structural analysis of peripheral airways and arteries in sudden fatal asthma. Am. Rev. Respir. Dis. 1991; 143: 138-143.

54. Kuwano K., Bosken C.H., Pare P.D. et al. Small airways dimensions in asthma and in chronic obstructive pulmonary disease. Am. Rev. Respir. Dis. 1993; 148: 1220-1225.

55. Haley K.J., Sunday M.E., Wiggs B.R. et al. Inflammatory cell distribution within and along asthmatic airways. Am. J. Respir. Crit. Care Med. 1998; 158: 565-572.

56. de Magalhäes Simöes S., dos Santos M.A. et al. Inflammatory cell mapping of the respiratory tract in fatal asthma. Clin. Exp. Allergy 2005; 35: 602-611.
57. de Medeiros Matsushita M., da Silva L.F., dos Santos M.A. et al. Airway proteoglycans are differentially altered in fatal asthma. J. Pathol. 2005; 207: 102-110.

58. Dolhnikoff M., da Silva L.F., de Araujo B.B. et al. The outer wall of small airways is a major site of remodeling in fatal asthma. J. Allergy Clin. Immunol. 2009; 123: 1090-1097.

59. Mauad T., Silva L.F., Santos M.A. et al. Abnormal alveolar attachments with decreased elastic fiber content in distal lung in fatal asthma. Am. J. Respir. Crit. Care Med. 2004; 170: 857-862.

60. Shiang C., Mauad T., Senhorini A. et al. Pulmonary periarterial inflammation in fatal asthma. Clin. Exp. Allergy 2009; 39: 1499-1507.

61. Hamid Q., Song Y., Kotsimbos T.C. et al. Inflammation of small airways in asthma. J. Allergy Clin. Immunol. 1997; 100: 44-51.

62. Hamid $Q$. Peripheral inflammation is more important than central inflammation. Respir. Med. 1997: 91 (Suppl. A): 11-12.

63. Kraft M., Djukanovic R., Wilson S. et al. Alveolar tissue inflammation in asthma. Am. J. Respir. Crit. Care Med. 1996; 154: 1505-1510.

64. McFadden E.R., Kiser R., DeGroot W.J. Acute bronchial asthma. Relations between clinical and physiologic measures. N. Engl. J. Med. 1973; 288: 221-225.

65. Kraft M., Pak J., Martin R.J. et al. Distal lung dysfunction at night in nocturnal asthma. Am. J. Respir. Crit. Care Med. 2001; 163: 1551-1556.

66. Lange P., Parner J., Vestbo J. et al. A 15-year follow-up study of ventilatory function in adults with asthma. N. Engl. J. Med. 1998; 339: 1194-1200.

67. Peat J.K., Woolcock A.J., Culle K. Rate of decline of lung function in subjects with asthma. Eur. J. Respir. Dis. 1987; 70: $171-179$.

68. in't Veen J.C., Beekman A.J., Bel E.H. et al. Recurrent exacerbations in severe asthma are associated with enhanced airway closure during stable episodes. Am. J. Respir. Crit. Care Med. 2000; 161: 1902-1906.

69. van Veen I.H., Sterk P.J., Schot R. et al. Alveolar nitric oxide versus measures of peripheral airway dysfunction in severe asthma. Eur. Respir. J. 2006; 27: 951-956

70. Leach C.L. Improved delivery of inhaled steroids to the large and small airways. Respir. Med. 1998; 92 (Suppl. A): 3-8.

71. Task Group on Lung Dynamics. Deposition and retention models for internal dosimetry of the human respiratory flow tract. Hlth Phys. 1966; 12: 173-208.

72. Lipworth B.J. Targets for inhaled drugs. Respir. Med. 2000; 94 (Suppl. D): S13-S16.

73. Leach C.L. The CFC to HFA transition and its impact on pulmonary drug development. Respir. Care 2005; 50: 1201-1206.

74. Leach C.L., Davidson P.J., Boudreau R.J. Improved airway targeting with the CFC-free HFA-beclomethasone metered-dose inhaler compared with CFC-beclomethasone. Eur. Respir. J. 1998; 12: 1346-1353.

75. Worth H., Muir J.F., Pieters W.R. Comparison of hydrofluoroalkane-beclomethasone dipropionate Autohaler with budesonide Turbuhaler in asthma control. Respiration 2001; 68: 517-526.

76. Reichel W., Dahl R., Ringdal N. et al. Extrafine beclomethasone dipropionate breath-actuated inhaler (400 micrograms / day) versus budesonide dry powder inhaler ( 800 micrograms / day) in asthma. Int. J. Clin. Pract. 2001; 55: 100-106.

77. Ederle K. Multicentre Study Group. Improved control of asthma symptoms with a reduced dose of HFA-BDP 
extrafine aerosol: an open-label, randomised study. Eur. Rev. Med. Pharmacol. Sci. 2003; 7: 45-55.

78. Davies R.J., Stampone P., O'Connor B.J. Hydrofluoroalkane-134a beclomethasone dipropionate extrafine aerosol provides equivalent asthma control to chlorofluorocarbon beclomethasone dipropionate at approximately half the total daily dose. Respir. Med. 1998; 92 (Suppl. A): 23-31.

79. Hampel F., Lisberg E., Guerin J.C. Effectiveness of low doses (50 and $100 \mu \mathrm{g}$ b.i.d) of beclomethasone dipropionate delivered as a CFC-free extrafine aerosol in adults with mild to moderate asthma. Study Group. J. Asthma 2000; 37: 389-398.

80. Fireman P., Prenner B.M., Vincken W. et al. Long-term safety and efficacy of a chlorofluorocarbon-free beclomethasone dipropionate extrafine aerosol. Ann. Allergy Asthma Immunol. 2001; 86: 557-565.

81. Fowler S.J., Currie G.P., Lipworth B.J. Step-down therapy with low-dose fluticasone-salmeterol combination or medium-dose hydrofluoroalkane 134a-beclomethasone alone. J. Allergy Clin. Immunol. 2002; 109: 929-935.

82. Lipworth B.J. The comparative safety / efficacy ratio of HFA-BDP. Respir. Med. 2000; 94 (Suppl. D): S21-S26.

83. Thongngarm T., Silkoff P.E., Kossack W.S., Nelson H.S. Hydrofluoroalkane-134A beclomethasone or chlorofluorocarbon fluticasone: effect on small airways in poorly controlled asthma. J. Asthma 2005; 42: 257-263.

84. Molimard M., Martinat Y., Rogeaux Y. et al. Improvement of asthma control with beclomethasone extrafine aerosol compared to fluticasone and budesonide. Respir. Med. 2005; 99: 770-778.

85. Busse W.W., Brazinsky S., Jacobson K. et al. Efficacy response of inhaled beclomethasone dipropionate in asthma is proportional to dose and is improved by formulation with a new propellant. J. Allergy Clin. Immunol. 1999; 104: 1215-1222.

86. Goldin J.G., Tashkin D.P., Kleerup E.C. et al. Comparative effects of hydrofluoroalkane and chlorofluorocarbon beclomethasone dipropionate inhalation on small airways: assessment with functional helical thin-section computed tomography. J. Allergy Clin. Immunol. 1999; 104: 258-267.

87. Hampel F., Lisberg E., Guerin J.C. Effectiveness of low doses (50 and $100 \mu \mathrm{g}$ b.i.d) of beclomethasone dipropionate delivered as a CFC-free extrafine aerosol in adults with mild to moderate asthma. Study Group. J. Asthma 2000: 37 (5): 389-398.
88. Ohbayashi H. One-year evaluation of the preventative effect of hydrofluoroalkane-beclomethasone dipropionate on eosinophilic inflammation of asthmatic peripheral airways. Respiration 2007: 74: 146-153.

89. Van Schayck C.P., Donnell D. The efficacy and safety of QVAR (hydrofluoroalkane-beclometasone diproprionate extrafine aerosol) in asthma (part 1): an update of clinical experience in adults. Int. J. Clin. Pract. 2004: 58: 678-688.

90. Dhillon S., Keating G.M. Beclometasone dipropionate / formotherol. In an HFA-propelled pressurized metered-dose inhaler. Drugs 2006; 66: 1475-1483.

91. Bousquet J., Poli G., Acerbi D. et al. Systemic exposure and implications for lung deposition with extra-fine HFA beclometasone dipropionat / formoterol fixed combination. Clin. Pharmacokinet. 2009; 48: 347-358.

92. De Backer W., Devolder A., Poli G. et al. Lung deposition of BDP / formoterol HFA pMDI in healthy volunteers, asthmatic, and COPD patients. J. Aerosol Med. Pulm. Drug Deliv. 2010; 23: 137-148.

93. Bonnet-Gonod F., Kottakis I., Ballabio M. et al. Superior efficacy of a low daily dose of a new fixed combination of beclometasone dipropionate / formoterol pMDI compared to an increased daily dose of BDP in moderate persistent asthma: a 3 month clinical study. $16^{\text {th }}$ Annual Congress of the European Respiratory Society; 2006 Sep. 2-6; Munich: P1237.

94. Huchon G., Magnussen H., Chuchalin A. et al. Lung function and asthma control with beclomethasone and formoterol in a single inhaler. Respir. Med. 2009; 103: 41-49.

95. Scichilone N., Battaglia S., Sorino C. et al. Effects of extrafine inhaled beclomethasone / formoterol on both large and small airways in asthma. Allergy 2010; 65: 897-902.

96. Papi A., Paggiaro P., Nicolini G. et al. Beclomethasone / formoterol vs fluticasone / salmeterol inhaled combination in moderate to severe asthma. Allergy 2007; 62: 1182-1188.

97. Papi A., Paggiaro P.L., Nicolini G. et al. Beclomethasone / formoterol versus budesonide / formoterol combination therapy in asthma. Eur. Respir. J. 2007; 29: 682-689.

\section{Информация об авторе}

Авдеев Сергей Николаевич - д. м. н., проф., руководитель клинического отдела ФГУ "НИИ пульмонологии" ФМБА России; тел: (495) 465-53-64; e-mail: serg_avdeev@list.ru

Поступила 14.12.10 С Авдеев С.Н., 2010 удК 616.248-092 


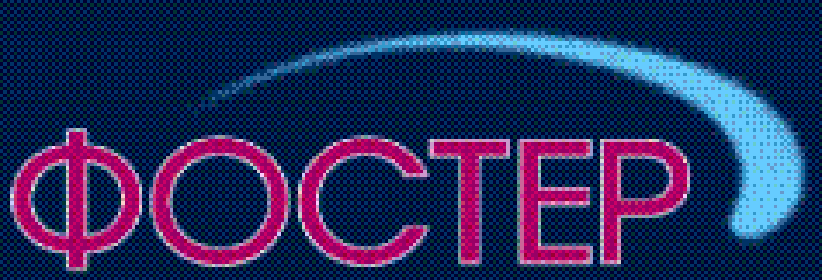

EeknomerazoH + Фopmorepon

\section{Первая экстрамелкодисперсная фиксированная комбинация ААя эффективного контроля бронхиальной астмы}

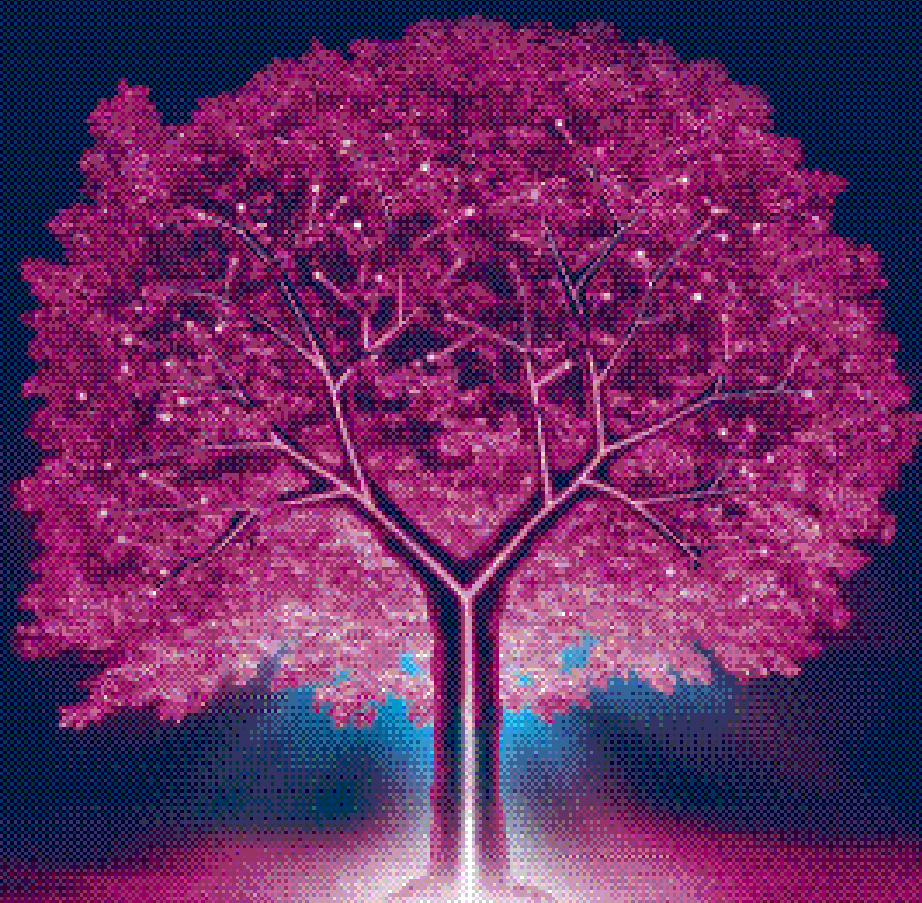

- Аостижение высокой легочной Аепозиции в центральных и периферических респираторных путях ${ }^{1-2}$

- Эффективное улучшение ФВА* и контроля наА СИмптомами бронхиаАьной астмы ${ }^{1}$

- Более быстрое начало Аействия по сравнению с фиксированной комбинацией Салметерол/ФАутиказон ${ }^{3}$

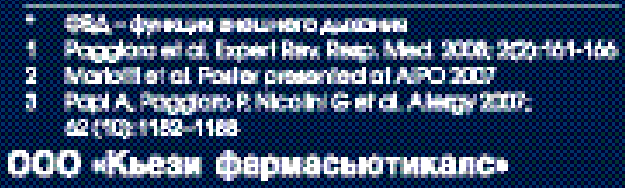

